\title{
Hombres que tienen sexo con hombres: conocimientos $y$ prácticas sexuales en relación a la epidemia del VIH/SIDA en Mérida, Yucatán, México.
}

\section{Artículo Original}

Ligia Vera-Gamboa, Brenda Cerón-Bracamonte, Renán A. Góngora-Biachi.

Universidad Autónoma de Yucatán, Centro de Investigaciones Regionales “Dr. Hideyo Noguchi”, Mérida, Yucatán, México.

RESUMEN.

INTRODUCCIÓN. En México la epidemia del VIH/ SIDA, mantiene un patrón predominante en la categoría homosexual/bisexual. A nivel nacional, Yucatán ocupa el primer lugar en esta categoría, con un $65 \%$, cifra superior al $54 \%$ del promedio nacional. OBJETIVO. Determinar los conocimientos, actitudes, prácticas sexuales, habilidades de prevención y percepción de riesgo en relación a la epidemia del VIH/SIDA, en un grupo de hombres que tienen sexo con otros hombres (HSH) en Mérida, Yucatán, México.

MATERIAL Y MÉTODO. Estudio descriptivo de junio de 1999 a octubre de 2000, en 50 HSH en la ciudad de Mérida, Yucatán, en quienes se aplicó un cuestionario estandarizado sobre conocimientos y actitudes sobre el VIH/SIDA, y posteriormente una entrevista semiestructurada.

RESULTADOS. Cerca del $100 \%$ de los $\mathrm{HSH}$ estudiados posee conocimientos sobre el VIH/SIDA, incluidos sus mecanismos de transmisión y prevención. El uso del condón lo reconocen como medida preventiva, pero sólo $70 \%$ refirió usarlo siempre. Los restantes no lo usan por confianza en su pareja. La mitad de ellos refiere preferencia por parejas sexuales masculinas y los restantes por hombres y mujeres. El $30 \%$ del grupo de HSH tenía una pareja femenina estable.

CONCLUSIONES. No existe solidez en los conocimientos sobre VIH/SIDA, lo que se traduce en prácticas de riesgo favorecedoras de la infección por el VIH, así como una baja percepción de riesgo. Se requieren campañas de prevención específicas para este grupo de población, con su participación, así como la creación de un entorno social de apoyo a través de campañas antidiscriminatorias.

(Rev Biomed 2004; 15:157-163)

Palabras clave: VIH/SIDA, hombres que tienen sexo con hombres, prácticas sexuales, campañas de

Solicitud de sobretiros: M.C. Ligia Vera Gamboa. Centro de Investigaciones Regionales "Dr. Hideyo Noguchi", Universidad Autónoma de Yucatán, Calle 59 No. 490 x Av. Itzáes Centro C.P. 97000, Mérida, Yucatán, México.

Tel: (52) (999) 928-41-57Ｅ-mail:vgamboa@tunku.uady.mx

Recibido el 16/Marzo/2004. Aceptado para publicación el 9/Julio/2004.

Este artículo está disponible en http://www.uady.mx/sitios/biomedic/revbiomed/pdf/rb041533.pdf 


\section{Vera-Gamboa, B Cerón-Bracamonte, RA Góngora-Biachi.}

prevención.

\section{SUMMARY.}

Men who have sex with men: knowleges and sexual practices in relation to HIV/AIDS epidemic, in Merida, Yucatan, Mexico.

Introduction. In Mexico, the HIV/AIDS epidemic maintains a prevailing pattern in the homosexual/ bisexual category. At the national level, Yucatan holds first place with $65 \%$ in this category, compared with the national average of $54 \%$.

Onjective. To determine the knowlege, attitudes, sexual practices, prevention, abilities, and risk perception in relation to the HIV/AIDS epidemic in a group of men who have sex with other men (MSM) in Merida, Yucatan, Mexico.

Methods and material. A descriptive study was carried out from June, 1999 to October, 2000 in 50 MSM, to whom a standardized questionnaire about knowledge and attitudes towards HIV/AIDS was administered, followed by a semistructured interview. Results. Almost 100\% of the MSM have knowledge about HIV/AIDS transmission including prevention mechanisms. Condom use was recognized as a preventive measure, but only $70 \%$ of the surveyed men referred to always use it, $30 \%$ of them don't use condom because they have confidence in their partner. Half of them referred to a preference for male sexual partners and the other half for male and female. Thirty percent of the MSM have a stable female sexual partner.

Conclusion. There is a lack of solidity of HIV/AIDS knowledge which is expressed as risk practices about HIV/AIDS infection and a low risk perception. Specific prevention campaigns for this group of the population with their participation, and the creation of a favorable social environment through non-discriminatory campaigns are required.

(Rev Biomed 2004; 15:157-163)

Key words: HIV/AIDS, men who have sex with men, sexual practices, prevention campaigns.

\section{ANTECEDENTES.}

Los nuevos casos de infección por el virus de inmunodeficiencia humana (VIH) ocurren en un $90 \%$ de los casos por contacto sexual sin protección (1). Así, la epidemia del Síndrome de Inmunodeficiencia Adquirida (SIDA), está condicionada por los comportamientos individuales que colocan a las personas en situaciones de riesgo para la infección por el VIH. Entre estos comportamientos se encuentran las relaciones sexuales entre varones, hecho presente en la mayoría de las sociedades, aunque a menudo, por diversos motivos, dichas relaciones son negadas y condenadas por la sociedad $(2,3)$. Esta negación y discriminación conducen a un grado variable de visibilidad pública del sexo entre hombres y a la clandestinidad de este tipo de relaciones, lo que incrementa la vulnerabilidad de este grupo hacia la infección por el VIH (2-5).

En diversas sociedades, la forma en que estos hombres consideran su identidad sexual, la determina el papel que desempeñan en el coito anal (6-9). Asimismo, existe un elevado porcentaje de HSH que son casados o mantienen relaciones sexuales también con mujeres. Este comportamiento bisexual parece ser frecuente en algunas sociedades, como es el caso de América Latina (2,10). Es importante mencionar también, que existen instituciones como, por ejemplo, el ejército y las cárceles, en las que los varones se ven obligados a pasar largas temporadas en compañía masculina exclusivamente y donde los comportamientos sexuales entre hombres pueden estar presentes (11). A nivel mundial, al menos de 5 a $10 \%$ de los casos de infección por VIH se deben a transmisión sexual entre varones $(2,10)$.

Los contactos sexuales entre varones incluyen coito anal, práctica con alto riesgo de transmisión de VIH tanto para el receptor como para el miembro activo, cuando no se utiliza condón. Debido a que la mucosa rectal puede lesionarse fácilmente con este tipo de práctica sexual y permitir de esta manera la entrada del virus. Además, ha sido sugerida la posibilidad de que la inmunidad natural al VIH de las células del revestimiento rectal, sea menor que la del revestimiento vaginal $(2,10,12)$. El contacto

\section{Revista Biomédica}


Prácticas sexuales en relación a la infección por VIH en HSH.

bucogenital también es común entre los HSH y si bien se había considerado más como un riesgo teórico, la evidencia actual en este tipo de práctica, señala que el riesgo debe considerarse (13).

Igualmente, la presencia de otras infecciones de transmisión sexual (ITS) no tratadas, como la sífilis, gonorrea o clamidiasis, pueden aumentar considerablemente el riesgo de transmisión del VIH, cuando éste también está presente (12,14-18).

En México, la epidemia del VIH/SIDA mantiene un patrón dominante de transmisión sexual y la categoría de transmisión homosexual/bisexual constituye la principal forma de transmisión con un 54\% (19, 20). En el estado de Yucatán, México, hasta Noviembre 2003, se habían registrado un total de 1661 casos . La categoría de transmisión principal entre los hombres, está en el grupo de práctica homosexual/ bisexual con 65\% de los casos (21), cifra superior a la media nacional en esta categoría (65\% vs 54\%).

Los objetivos del presente trabajo fueron: determinar los conocimientos, actitudes y prácticas sexuales, así como las habilidades de prevención y percepción de riesgo en relación a la epidemia del VIH/SIDA, en un grupo de HSH en Mérida, Yucatán, México.

\section{MATERIAL Y MÉTODOS.}

Se realizó un estudio descriptivo de Junio de 1999 a Octubre de 2000. Al desconocerse el número de HSH, se trabajó con una muestra no probabilística. A través de informantes clave de la comunidad de HSH se realizó una invitación a integrantes de este grupo, respondiendo a la invitación $50 \mathrm{HSH}$.

Previo consentimiento informado por escrito, la información se obtuvo a través de un cuestionario estandarizado sobre conocimientos y actitudes ante el Sida (22). Posteriormente, se realizó a cada participante una entrevista semistructurada para conocer sobre sus prácticas sexuales, habilidades de prevención y percepción de riesgo para la infección por VIH.

\section{RESULTADOS.}

La edad promedio del grupo de HSH fue de 27.4 años (intervalo 18-59), de ellos el 30\% vivía conyugalmente con una pareja femenina (cuadro 1).

\section{Cuadro 1}

Algunas características sociodemográficas de hombres que tienen sexo con hombres en Mérida, Yucatán (N=50).

\begin{tabular}{lc}
\hline Estado civil & n y porcentaje \\
Soltero & $35(70 \%)$ \\
Casado & $13(26 \%)$ \\
Unión libre & $2(4 \%)$ \\
\hline Escolaridad & \\
$0-5$ años & $2(4 \%)$ \\
$6-9$ años & $10(20 \%)$ \\
10 o + & $38(76 \%)$ \\
\hline
\end{tabular}

En relación a los conocimientos sobre la infección del VIH, el 98\% refirió el concepto de que la infección por el VIH es de transmisión sexual (ITS) que destruye las defensas del organismo, y sólo uno (2\%), refirió un concepto poco claro pero sin ubicarlo en el plano religioso o compararlo con otro padecimiento. Aunque el $100 \%$ de los encuestados reconoce que cualquier persona, sin importar sexo, raza, edad, religión o condición socioeconómica, puede infectarse, se encontró que aún existen algunos conceptos equivocados en relación a esta infección (cuadro 2). Dentro de los mecanismos de transmisión señalados, el $100 \%$ reconoce la vía sexual y sanguínea como mecanismos de transmisión, pero también señalaron otras formas de transmisión que no lo son, como picaduras de insectos. En cuanto al conocimiento de las medidas de prevención, el $30 \%$ consideró los exámenes periódicos y el $22 \%$ la selección del compañero sexual como formas de prevención (cuadro 2).

En cuanto al uso del condón, el 100\% de los encuestados reconoce que su uso previene contra la infección, pero su conocimiento en relación a su uso correcto y en el momento oportuno aún es insuficiente, ya que sólo el $70 \%$ de ellos refirió que debe utilizarse 
L Vera-Gamboa, B Cerón-Bracamonte, RA Góngora-Biachi.

Cuadro 2

Medidas de prevención señaladas por hombres que tienen sexo con hombres en Mérida, Yucatán, México (N=50).

\begin{tabular}{lccc}
\hline Medidas de prevención & Sí es & No es & Lo Desconocen \\
\hline Evitar practicas sexuales de riesgo & $49(98 \%)$ & 0 & $1(2 \%)$ \\
Utilizar agujas nuevas & $47(94 \%)$ & $2(4 \%)$ & $1(2 \%)$ \\
Fidelidad & $46(92 \%)$ & $2(4 \%)$ & $2(4 \%)$ \\
Abstinencia sexual & $43(86 \%)$ & $6(12 \%)$ & $1(2 \%)$ \\
Selección del compañero sexual & $38(76 \%)$ & $11(22 \%)$ & $1(2 \%)$ \\
Higiene esmerada & $28(56 \%)$ & $19(38 \%)$ & $3(6 \%)$ \\
Aislar a los infectados & $18(36 \%)$ & $30(60 \%)$ & $2(4 \%)$ \\
Examen médico periódico & $15(30 \%)$ & $32(64 \%)$ & $3(6 \%)$ \\
Ingesta de medicamentos & $11(22 \%)$ & $35(70 \%)$ & $4(8 \%)$ \\
\hline
\end{tabular}

siempre que se tengan relaciones, independientemente de con quién se tengan éstas. Igualmente, en cuanto al uso, refirieron la utilización de cremas o vaselina (73\%) o bien que el empaque del condón puede abrirse con las uñas, dientes o tijeras (88\%).

Por lo que respecta a las actitudes, respecto a la conducta que adoptarían al enterarse de que otra persona estuviera infectada por el VIH, la mitad de ellos le brindaría su apoyo, independientemente de que se tratara de un amigo, familiar o su pareja. El 96\% de los encuestados refirió que les gustaría tener mayor información acerca de la infección y su prevención

En relación a las prácticas sexuales encontramos que en cuanto al número de parejas sexuales en el último mes, el 38\% (19/50) refirió haber tenido dos o más y un $18 \%$ refirió encuentros sexuales con parejas casuales y en cuanto al género de sus parejas, el $50 \%$ de los encuestados refirió preferencia por el género masculino y el otro $50 \%$ refirió preferencia tanto por hombres como por mujeres.

Se encontró que la práctica sexual realizada con mayor frecuencia, era aquella que mayor placer les producía a ellos y a su pareja, y que estas prácticas no eran excluyentes entre sí (figura 1). Asimismo, el papel más frecuente adoptado durante las prácticas sexuales fue el receptivo, aunque un $24 \%$ refirió prácticas insertivo-receptivas, esto es, prácticas mixtas Revista Biomédica (figura 2).

Cuando se investigó si habían hablado acerca de la infección con su pareja, la mayoría (39/50), refirió haberlo hecho, porque pensaban que era una forma de prevenir; 4/50 (8\%), estaba de acuerdo en hablar con su pareja con respecto a la infección por VIH/ SIDA, no habiéndolo realizado hasta el momento, porque no se consideraban en riesgo o porque tenían confianza en su pareja. El catorce por ciento (7/50) indicó que no hablaría con su pareja porque no tenían riesgo.

Al preguntar sobre el uso del condón y la negociación de su uso, 31(62\%) dijo que siempre lo usaban cuando desconfiaban o se sentían en riesgo; 6

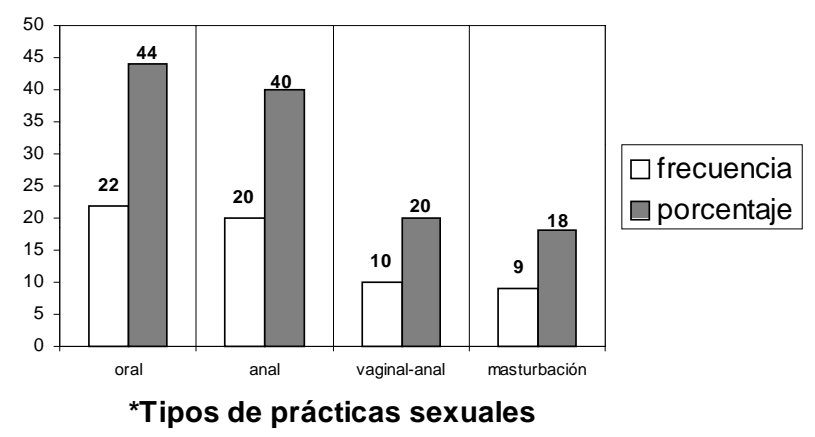

*Nota.- Las prácticas sexuales no son excluyentes entre sí.

Figura 1.- Prácticas sexuales más frecuentes referidas por 50 hombres que tienen sexo con hombres en Mérida, Yucatán, México. 


\section{Prácticas sexuales en relación a la infección por VIH en HSH.}

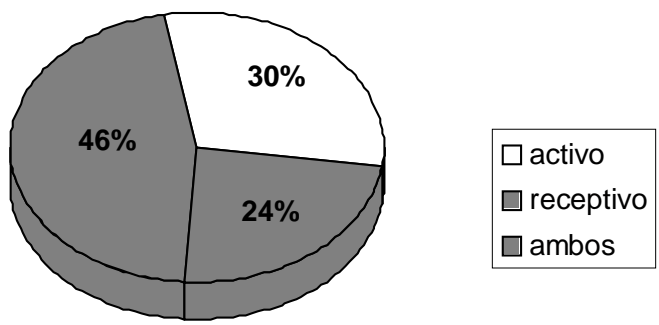

Figura 2.- Rol durante la práctica sexual de $50 \mathrm{HSH}$ de Mérida, Yucatán, México.

(12\%) dijeron que sí lo negociarían si lo consideraran necesario y 13 (26\%) refirieron innecesario el uso del condón pues confiaban en sus parejas. A cada uno de los participantes se le preguntó si se consideraba en riesgo para la infección y cómo lo clasificarían. Sus respuestas quedaron de la siguiente manera: $8(16 \%)$ no se considera en riesgo, $22(44 \%)$ respondieron considerarse en un riesgo bajo, sólo 10 (20\%) estimaron estar en un alto riesgo para la infección por el VIH y los restantes (20\%) no respondieron. Acerca de su opinión sobre si existen campañas específicas para los HSH, 21 (42\%) opinaron que no, 3 (6\%) refieren que sí existen pero falta darles difusión, 5 (10\%) refiere que probablemente sí existen pero las desconocen y 21 (42\%) refiere que sí existen.

\section{DISCUSIÓN.}

Aunque prácticamente el $100 \%$ de los encuestados tiene conocimientos sobre el Sida, aún existen conceptos poco claros relacionados con los mecanismos de infección y los métodos de prevención para la infección por VIH/SIDA en el grupo estudiado. Esto puede deberse a que la relacionan con las otras ITS y a conjeturas elaboradas con base en conceptos erróneos o poco claros, como lo hacen suponer ciertas preguntas y comentarios vertidos por ellos en la entrevista efectuada a cada individuo. Por ejemplo, considerar la picadura de insectos como vía de transmisión o los exámenes periódicos como medida preventiva. Es posible que estas mismas carencias o falta de solidez en el conocimiento de la temática los lleve al inadecuado uso del condón y a no platicar con sus parejas sobre el tema de transmisión y prevención de la infección por VIH/SIDA $(14,23)$.
Es importante mencionar que el $22 \%$ de ellos considera innecesario el hecho de platicar con su pareja sexual acerca del VIH/SIDA porque no se percibe en riesgo. Esto último, principalmente por confianza en su pareja; en particular si ésta es femenina. Sin embargo, el 50\% de la población entrevistada realiza prácticas bisexuales y lo confirma el hecho de que el $30 \%$ de ellos tenían al momento del estudio una pareja femenina estable. Esta situación, que se conoce como el principal mecanismo de transmisión de la infección por VIH en la población femenina en Yucatán (24), es un elemento a considerar en las campañas de prevención dirigidas a los $\mathrm{HSH}$ y a las mujeres.

Asimismo, hay que señalar que las prácticas mixtas (insertivo-receptivas) fueron las predominantes en este grupo de HSH (46\%), hecho que se ha asociado a un mayor riesgo para la infección por VIH (25-27).

Aquellos que refirieron prácticas sexuales con parejas ocasionales (18\%) manifestaron que éstas fueron en un momento de "apasionamiento o calentura" y no recordaron el uso del condón, o si lo recordaron, prefirieron no mencionarlo para no despertar desconfianza en la pareja. Situación similar se presentó con el hecho de hablar sobre el tema, hecho ya señalado para la baja percepción de riesgo para la infección. Datos semejantes fueron encontrados en un grupo de HSH de Popolnah, una comunidad rural de Yucatán, México (28).

Resalta el hecho de que las prácticas sexuales de los HSH de este estudio, poco han cambiado en relación con los reportes de los primeros estudios en estos grupos de población (14, 25-27).

Con relación a las campañas de prevención, llama la atención que el 32\% de la población entrevistada refiere no conocer alguna campaña de prevención de VIH y con relación a si existen campañas específicas para el grupo de HSH, el $42 \%$ refiere que no existen. Es un hecho que las campañas actuales van enfocadas hacia la población heterosexual y algunos promocionales fomentan la prevención en la figura femenina, lo que puede producir en los HSH la sensación de que ellos no corren riesgo y con ello 


\section{Vera-Gamboa, B Cerón-Bracamonte, RA Góngora-Biachi.}

concluir que no es prioritario protegerse.

Considerando que los HSH son el grupo principalmente afectado en Yucatán, México, y que Mérida es el epicentro de la epidemia (24), nuestros resultados apoyan la idea de la elaboración de programas de educación para la prevención de infección por VIH específicos para los $\mathrm{HSH}$, que promuevan el desarrollo de actitudes y conductas y les permitan protegerse de esta infección retroviral. Asimismo, se precisan políticas de promoción, prevención y apoyo dirigidas hacia y para los $\mathrm{HSH}$, considerando su participación y la creación de un entorno social favorable para la atención y prevención de la infección del VIH, a través de campañas no discriminatorias, como ha sido recomendado por ONUSIDA (2) desde 1998.

\section{AGRADECIMIENTOS.}

Agradecemos la valiosa colaboración del C.P. Demetrio Burgos Domínguez, representante regional del Frente Nacional de Personas Afectadas por el VIH (FRENPAVIH), para poder establecer contacto con las personas que participaron en el presente estudio.

\section{REFERENCIAS.}

1.- Idemyor V. Promising microbicide approach for prevention of human inmodeficiency virus transmission needs more support. Pharmacotherapy 2002; 22: 1074-6.

2.- Anónimo. El SIDA y las relaciones sexuales entre varones. Punto de vista. Julio 1998. Programa Conjunto de las Naciones Unidas sobre el VIH/SIDA. Ginebra, Suiza, 1999: p: 1-8.

3.- Consulta Regional sobre programas de prevención, atención y apoyo en VIH/SIDA a hombres que tienen sexo con hombres en América Latina y el Caribe. Colección Prácticas Óptimas. Programa Conjunto de las Naciones Unidas sobre el VIH/SIDA. Ginebra, Suiza, 1999: p: 1-24.

4.- Anónimo. Intervenciones en VIH/SIDA en hombres que tienen sexo con hombres. Family Health International. Virginia, USA. Junio 2001. Disponible www.fhi.org Noviembre 2003.

5.- Toro-Alfonso J. Trabajo en la promoción de salud en una comunidad de trabajadores sexuales en San Juan, Puerto Rico y la prevención del VIH. Avances en Psicología Clínica Latinoamericana 1995; 13:55-70.

\section{Revista Biomédica}

6.- Carrier JM. Participants in urban Mexican male homosexual encounters. Arch of Sex Behavior 1971; 1: 279-291.

7.- Carrier JM, Mexican male bisexuality. J Homosexuality. 1985;11:75-85.

8.- Izazola-Licea JA. La bisexualidad. En: Antología de la Sexualidad Humana. CONAPO. $1^{\mathrm{a}}$. Ed. Ed Porrua, México 1994. p. 633-67.

9.- Izazola JA, Astarloa L, Beloqui J, Brofman M, Cheuqer P, Zacarías F. Avances en la comprensión del VIH/SIDA: una visión multidisciplinaria. En: J.A. Izazola Ed. El Sida en América Latina y el Caribe: una visión multidisciplinaria. Fundación Mexicana para la Salud. México. 1999. p. 21-46

10.- Anónimo. El Sida y las relaciones sexuales entre varones. Documento de actualización técnica. Colección Prácticas Óptimas del ONUSIDA. Ginebra, Suiza, Mayo de 2000. p. 18.

11.- Anónimo. Young men at special risk. En: young men and HIV. Culture, poverty, and sexual risk. Ed. Joint United Nations Programme on HIV/AIDS/The Panos Institute. 2001. p. 23-9.

12.- Fauci A, Lane C. Transmisión del virus de inmunodeficiencia humana. En: Isselbacher K, Braunwald E, Wilson J, Fauci A, Kasper D. Ed. Principios de Medicina Interna 13a. Ed. Interamericana. México. Mc Graw Hill 1994. p. 1802-58.

13.- Vera-Gamboa L. Rutas de infección por el virus de inmunodeficiencia humana inicialmente no consideradas factibles. Rev Biomed 2001; 12:122-9.

14.- Laga M, Alary M, Nzila N, Manoka AT, Tuliza M, Behets F et al. Condom promotion, sexually transmitted disesases treatment, and declining incidence of HIV-1 infection in female Zairian sex workers. Lancet 1994; 344: 246-8.

15.- Del Rio-Chiriboga C, Rico B. Prevención de la transmisión sexual: evaluación y perspectivas. SIDA/ETS 1996; 2:47-53.

16.- Terán X. Enfermedades de transmisión sexual. SIDA/ ETS 1997; 2: 141-6.

17.- Rico B, Liguori AL. Mujeres y VIH/SIDA: reflexiones sobre la situación actual y algunos retos legislativos. SIDA/ ETS 1998; 4: 40-7. 
Prácticas sexuales en relación a la infección por VIH en HSH.

18.-Fleming DT, Wasserheit JN. From epidemiological synergy to public health policy and practice: the contribution of other sexually transmitted diseases to sexual transmission of HIV infection. Sex Transm Infect 1999; 75: 3-17.

19.- Epidemiología del VIH/SIDA en México en el año 2003. Datos al 1 de noviembre 2003. Disponible en: www.salud.gob.mx/conasida Revisado en Diciembre 2003.

20.- Magis-Rodríguez C, Bravo-García E, Uribe-Zuñiga P. Dos décadas de la epidemia del Sida en México. Disponible en: www.salud.gob.mx/conasida Revisado en Diciembre 2003.

21.- Servicios de Salud de Yucatán. Departamento de Vigilancia epidemiológica. Programa de Prevención y Control del VIH/SIDA. Noviembre 2002.

22.- Castro-Sansores C, López-Avila MT, Góngora-Biachi RA. Conocimientos e impacto de tres medios de enseñanza en relación al Síndrome de Inmunodeficiencia Adquirida. Rev Biomed 2000; 11:7-16.

23.- Agleton P. Ya se dispone de antiretrovíricos contra el VIH/SIDA: ¿ es necesaria ahora la prevención? Salud Pública Méx. 2000; 25: 197-204.

24.- Góngora-Biachi RA. La infección del VIH y el Sida en Yucatán: un análisis situacional basado en la investigación. Enf Inf Microbiol 2000; 20:207-13.

25.- Hernández M, Uribe P, Gortmaker S, Avila C, De Caso LE, Muller N, et al. Sexual behavior and status for human immunodeficiency virus type 1 among homosexual and bisexual males in México city. Am J Epidemiol 1992; 135:88394.

26.- Cáceres CF, Van Griensven GJP. Male homosexual transmisión of HIV-1. AIDS 1994; 8:1051-61.

27.- Izazola-Licea JA, Avila-Figueroa C, Gortmaker SL, Del Río-Chiriboga C, Transmisión homosexual del VIH/SIDA en México. Salud Pública Méx. 1995; 37: 602-14.

28.- Vera-Gamboa L, Cen-Chablé M, Góngora-Biachi RA. VIH/ SIDA. Conocimientos, actitudes y prácticas sexuales en un grupo de hombres que tienen sexo con hombres en una comunidad rural de Yucatán, México. Arch Hispanoamer Sex 2003;9: 191-202. 